Article

\title{
An Adaptive Routing-Forwarding Control Scheme Based on an Intelligent Fuzzy Decision-Making System for Opportunistic Social Networks
}

\author{
Yian Zhu ${ }^{1}$, Lin Zhang ${ }^{1, *}$, Haobin Shi ${ }^{1,2}$, Kao-Shing Hwang ${ }^{3, *}$, Xianchen Shi ${ }^{1}$ and Shuyan Luo ${ }^{1}$ \\ 1 School of Computer Science, Northwestern Polytechnical University, Xi'an 710129, China \\ 2 Key Laboratory of Big Data Storage and Management, Ministry of Industry and Information Technology, \\ Northwestern Polytechnical University, Xi'an 710072, China \\ 3 Department of Electrical Engineering, National Sun Yat-sen University, Kaohsiung 80424, Taiwan \\ * Correspondence: zhanglin_npu@outlook.com (L.Z.); hwang@mail.ee.nsysu.edu.tw (K.-S.H.)
}

Received: 8 August 2019 ; Accepted: 28 August 2019 ; Published: 2 September 2019

\begin{abstract}
Routing selection in opportunistic social networks is a complex and challenging issue due to intermittent communication connections among mobile devices and dynamic network topologies. The structural characteristics of opportunistic social networks indicate that the social attributes of mobile nodes play a significant role on data dissemination. To this end, in this paper, we propose an adaptive routing-forwarding control scheme (FPRDM) based on an intelligent fuzzy decision-making system. On the foundation of the conception of fuzzy inference logic, two techniques are used in the proposed routing algorithm. Information fusion of social characteristics of message users and node identification are implemented based on the fuzzy recognition strategy, and the fuzzy decision-making mechanism is applied to control message replication and optimize data transmission. Simulation results demonstrate that, in the best case, the proposed scheme presents an average delivery ratio of 0.8 , reduces the average end-to-end delay by nearly $45 \%$ as compared with the Epidemic routing protocol, and lowers the network overhead by about $75 \%$ as compared to the Spray and Wait routing algorithm.
\end{abstract}

Keywords: opportunistic social networks; routing algorithm; fuzzy inference logic; intelligent fuzzy decision-making system; data dissemination

\section{Introduction}

Opportunistic social networks (OSNs) [1,2], a type of complex intermittently connected network architecture with a foundation in opportunistic communication and node profiles, have emerged from both a type of delay tolerant network (DTN) [3] and social network service (SNS) [4], and it has been considered a promising network model to improve data transmission reliability. Additionally, the structure in OSNs is characterized by several features such as node mobility, network topology, and social attributes [5]. As reliable and continuous data connectivity can not be available in OSNs, nodes in unpredictable online communities typically take the opportunity of encountering the peers created by their social attributes to communicate with each other at unpredictable intervals [6]. In recent years, the research interests in OSNs mainly consist of the routing-forwarding approach [5], mobility patterns [7], security with privacy [8], and data storage and management [9].

With the increasing expansion of 5G networks and big data environments [10], the high-quality requirements for mass-data multiprocessing have led to a tremendous growth in the number of smart mobile devices, such as Bluetooth devices, smart phones, laptops or other wearable sensor devices [10]. This is more conducive for the construction of opportunistic mobile social networks in urban or remote areas [11]. Moreover, the dawn of big data environments and 5G networks means that the 
high-fast, low-consumption and low-delay data transmission mode requires higher storage spaces and processing capacity from smart mobile devices. The data packets circulating in peer-to-peer communication may be text messages, pictures, or files such as video that occupy relatively large cache spaces [12]. However, due to the insufficient storage spaces and relatively low processing capacity of smart mobile devices, it is particularly difficult for nodes to implement the peer-to-peer communication between message applications in $5 \mathrm{G}$ networks and big data environments. Therefore, opportunistic mobile social networks propose that an end-to-end communication connectivity can be established using several suitable relay nodes to carry out the store-carry-forward mechanism [13], which indicates mobile nodes with smart devices temporarily store data packets in their buffer memory and carry them until they encounter other suitable relay nodes to forward these data packets. Therefore it is extremely important for a node in OSNs to select suitable next hop nodes for data dissemination [14].

As various applicable routing-forwarding strategies [15] have been proposed to tackle the problem of data dissemination for different scenarios in OSNs, most of them make an accurate message delivery decision by comprehensively assessing reliable social information associated with nodes such as social features [6], human mobility [1], contact history [13], or level of credibility. As a general rule, the social features of humans, which can be easily extracted from portable mobile devices, are the most widely utilized metric information for relay node selection in OSNs. On the one hand, as the main carriers of mobile devices, humans in opportunistic mobile social networks commonly present multiple different social attributes, even including several significant features that facilitate the data transmission process [16]; on the other hand, it is practicable to implement the recognition of relay nodes or destinations via the social features of humans in OSNs [17].

Additionally, how to utilize the effective social features of humans to implement an efficient data dissemination process between source nodes and destinations, is one of the most long-standing and elusive challenges in OSNs. With the aim of yielding low overhead, low network delay, and high delivery ratio, multitudinous routing approaches employ various strategies to create a better data dissemination environment in the OSNs. As one of the dominant algorithms, flooding-based routing protocols [18] transmit message replications to every node that the carriers encounter, which causes the overspread of data packets and a huge waste of cache spaces. Then, several routing protocols have been proposed to reduce the high overhead through controlling the number of message copies. Moreover, there are many other routing protocols based on message replication [19], direct delivery [20], mobility prediction [21], phase division [22], and probability evaluation [23] that have been utilized in the OSNs. Meanwhile, profile-based routing protocols employ different social attributes of mobile users such as spatial and temporal mobility features, social bonds, social influence, or node similarity, to enhance transmission efficiency in the OSNs, which mainly focus on single or multiple attribute characteristics of message users such as hobbies and interests, selfish behavior, encounter history, or distance and location of node mobility [24-26].

However, in the social scenarios of opportunistic mobile social networks, there are still many challenges for these routing methods that have not been well addressed: (1) It is challenging to construct an applicable mathematical framework to comprehensively consider the numerous attribute features of mobile users, including long-term stability characteristics and short-term variable characteristics, such as occupation, appearance, location or online community [27]. (2) Because each attribute feature of mobile users plays a different role in the process of message routing and forwarding, how to dynamically allocate a reasonable weight value to them is another key issue to be tackled [28].

To address these open questions, we propose and develop an adaptive routing-forwarding control scheme based on fuzzy pattern recognition and decision-making system (FPRDM) for opportunistic social networks, which mainly consists of four phases: fuzzy recognition for node classification, weight adjustment for social features, fuzzy relationship inference, and fuzzy decision-making process. To be specific, after the social attributes of mobile users are formalized, quantization vectors of these features will be regarded as fuzz input values [29] that are adopted to recognize node classification (relay node or irrelevant node). Then, this approach utilizes the improved analytic hierarchy process 
(AHP) [30] to allocate a seemly weight value to each fuzzy input, thereby determining the fuzzy mathematical mapping of the social features of mobile users into a transmission priority value. Eventually, the message routing-forwarding process will be performed via a feedback mechanism and a defuzzification component. On the whole, the main contributions of this paper are listed as follows:

- A fuzzy inference model is proposed to implement the fusion of multiple social information of mobile users, thereby providing a reliable and stable strategy for opportunistic message routing and forwarding.

- To synthetically evaluate the impact of each social characteristic on the data transmission process in OSNs, we combine the fuzzy inference logic with the analytic hierarchy process, and more importantly, with exploring the data transmission relationships among mobile users.

- On the basis of a feedback mechanism, we are able to build a relatively stable and sustained data transmission connectivity between the source nodes and destinations in opportunistic mobile social network environments.

- Ultimately, simulation results demonstrate that this scheme reduces the network delay and the overhead ratio, and enhances the delivery ratio as compared to several other typical or latest routing protocols in the OSNs.

As a supplement to routing algorithms in multi-layer social networks, this FPRDM mechanism constructs an effective communication link between nodes in different online communities or the same online community by transforming the social attribute characteristics of nodes into a strong basis for data dissemination.

\section{Related Works}

In recent several years, for the sake of the construction of a efficient data transmission connectivity between source node and destination, more and more routing algorithms [5] which try using new techniques and theories have been proposed for different application scenarios in the OSNs. The routing protocols that utilize node profile to establish the data transmission connectivity are called profile-aware algorithms. On the contrary, the routing approaches that do not employ node profiles to perform the data transmission process are profile-ignorant algorithms [27]. We will introduce the two categories of routing algorithms related to our works in detail.

\subsection{The Proposed Profile-Aware Routing Algorithms for Opportunistic Mobile Social Networks}

In opportunistic mobile social networks, the Epidemic routing protocol [31] is commonly regarded as a benchmark for most routing strategies because of its high delivery ratio and network delay. For this reason, Halikul L and Mohamad A [2] introduced a social-based Epidemic-based routing protocol (EpSoc). This approach adopts a significant social feature of mobile users which is degree centrality to effectively control the number of message replications. To improve the data dissemination environment, K. Liu et al. [6] proposed a fuzzy routing-forwarding approach, which utilizes a type of comprehensive node similarity that is the combination of mobile and social similarities to make an accurate decision on relay node selection and message forwarding. Borrego $C$ et al. [22] recommended a composite routing-delivery scheme, namely explore and wait, for node profile-casting in OSNs. In the explore phase, a profile-based model that allows data packets to be transmitted to profiles that are defined by a delivery function; and message carriers wait until it encounters a node that satisfies the delivery function and then sends data packets to the node in the wait phase.

Additionally, the social relationship and features of mobile users could be exploited to improve the performance of message delivery. Yang $Y$ et al. [32] introduced a routing protocol called the Geo-Social-Interest algorithm, which fully considers the impact of geographical information, social characteristics and node interests and then implements a complete data transmission connectivity. Q. Xu et al. [33] developed a routing algorithm on the grounds of Epidemic information dissemination, which defines the social characteristics of mobile users as pre-immunity and immunity, establishing an 
analytical system by ordinary different formulas to mimic Epidemic information transmission in OSNs. In addition, $\mathrm{Li} \mathrm{N}$ et al. [34] proposed a cross-layer and reliable routing protocol that utilizes fuzzy inference logic and network topology control strategies to enhance the efficiency and the reliability of message routing and forwarding in mobile ad hoc networks.

Moreover, in the multi-layer opportunistic social networks [35], the routing algorithm based on social community division employs some different real mobility traces to evaluate their performance. Magnani et al. [36] introduced a novel algorithm model for multi-layer opportunistic social networks, where mobile users belong to different types of networks in the same time, and the SNA (social network analysis) centrality metrics have been extended in a real mobility dataset. Additionally, Q. Xu et al. [33] developed a novel analytical model to assess the Epidemic data dissemination in mobile opportunistic social networks. This algorithm adopts two novel elements (per-immunity and immunity) to evaluate the change of mobile nodes' interests. Through experimental evaluation, this analytical model based on users' behaviors and interests outperforms other existing ones.

\subsection{The Proposed Profile-Ignorant Routing Algorithms for Opportunistic Mobile Social Networks}

To improve the delivery ratio and reduce the end-to-end delay and the network overhead, the profile-ignorant routing approaches in OSNs commonly prefer to try using many new mathematical methods and theories. J. $\mathrm{Wu}$ [9] designed a cache management routing algorithm, and a node extend routing algorithm, which respectively utilize probability prediction, and node cooperation to improve the data transmission environment. Additionally, Luo J et al. [17] recommended an opportunistic routing algorithm that employs optimization methods and distance estimation to control the number of relay nodes. Prodhan A T et al. [18] introduced a new quota-based routing algorithm, which increases the chance of message forwarding by evaluating the transmission priority of the data packet that has the earliest deadline. Wang, Y et al. [28] developed a novel cooperative store-carry-forward method to decrease the parking time of the vehicle in dark areas, utilizing bidirectional vehicle streams and selecting two vehicles in both directions for the target vehicle.

In addition, many mathematical methods such as graph theory [37], Markov chain [38] or information entropy [39] have been utilized to implement the combination of node recognition and data transmission in opportunistic mobile social networks. Moreover, the fuzzy inference logic [40], which is capable of converting numerical or textual input vectors into an evaluation fuzzy subset [29], is a novel and feasible approach in the research field of opportunistic routing and forwarding, establishing a nonlinear or linear mathematical mapping from the social features of mobile users to the metric values of data dissemination [41]. Based on that, message carriers are able to make more reasonable next-hop node selections and message delivery decisions in the OSNs [6].

Compared with these existing algorithms, our work mainly focuses on the data dissemination relationship between mobile nodes, which means that this scheme transforms the social behaviors and characteristics of nodes into the data transmission association between them. That also becomes a strong basis for information routing and forwarding. Besides, we transform the social connection between nodes into the fuzzy relation of data transmission through the method of fuzzy inference logic, and evaluate the data transmission relationship between mobile users through the defuzziness.

\section{System Model Design}

Fuzzy recognition and decision-making systems [40], novel adaptive techniques with a foundation in fuzzy inference and mathematics, are emerging in recent years as a decision support system, promising to be applied to provide more accurate decisions for routing selection, and data transmission in 5G networks, and big data environments. Because the relationship between nodes' transmission characteristics and their social attributes is fuzzy and uncertain, we attempt to employ a fuzzy inference system [41] to evaluate their importance on data transmission, thereby establishing a more efficient dissemination process. Then, we develop an adaptive routing-forwarding model exploiting fuzzy recognition and decision-making for OSNs. 


\subsection{The Overall Structure of Intelligent Fuzzy Decision-Making System for Opportunistic Mobile Social Networks}

Referring to the classical applied fuzzy control system [29], the overall structure of the fuzzy recognition and decision-making model for opportunistic mobile social networks can be divided into five successive phases, including information acquisition, data processing and feature extraction, pattern matching and model building, model training and testing, and decision-making. Because the operational processes in this system are independent of each other, message application carriers in the OSNs are able to gather, collate and manage a large amount of effective information from multiple different neighbor nodes synchronously. Next, the detailed description of each procedure (as shown in Figure 1) in this system is summarized as follows:

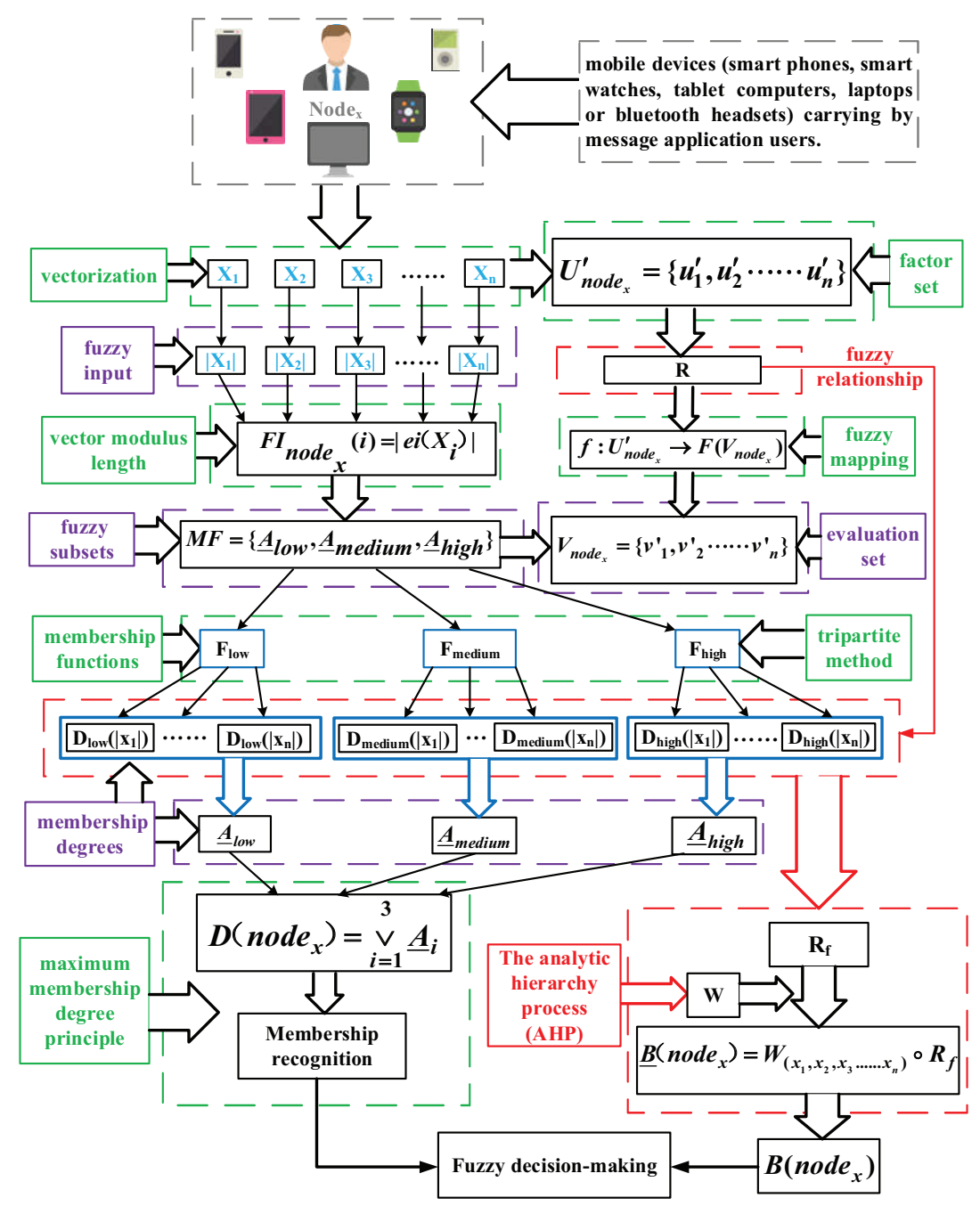

Figure 1. The overall structure of fuzzy recognition and decision-making model for opportunistic social networks.

As shown in Figure 1, message application users with mobile high-tech devices (smart phones, smart watches, tablet computers, laptops or Bluetooth headsets) in different online communities of opportunistic mobile social networks commonly present multiple disparate social characteristics, even including many significant features that facilitate the data transmission process. Consequently, several important social attributes of nodes will be collected by this system and could be adopted to evaluate the special social relationship between destination and relay nodes, which is especially conducive to establishing a sustaining and stable data transmission link. 
Then, all of the data associated with social attributes, obtained from neighbor nodes, are suitably processed from irregular and unrecognizable to regular and identifiable formats. The process of pattern matching and model building is scrupulously divided into two parts: The course of membership recognition for nodes, and the course of fuzzy relationship inference. Each membership function is determined based on the guidelines of the tripartite method. According to the concept of the intersection membership method, three different levels of a single membership degree could be generated from different membership degrees, and afterwards the final fuzzy control result is obtained from three different levels of single membership degrees on the basis of maximum membership principle.

Afterwards, weight adjustment for the fuzzy relationship assessment matrix and data training are implemented based on the analytic hierarchy process (AHP) [30]. As shown in Figure 1, the weight matrix is gradually determined thorough the AHP after multiple systematic and manual parameter evaluations. In addition, the inference result of the fuzzy relationship will be calculated through the weight matrix and the fuzzy relationship assessment matrix. Ultimately, the processes of data fusion and data transmission decision-making are performed in this system. Message application users constantly collect and compare the transmission value of message application users, comprehensively assess the attributive characters of these neighbor nodes, and come to an accurate decision on data dissemination based on the feedback mechanism.

\subsection{Fuzzy Pattern Recognition Process for Node Classification in Opportunistic Mobile Social Networks}

From different online communities of opportunistic social networks, message application users carrying high-tech devices must present some different actions, such as executing conversations, subscribing to information, interactions and mutual following, posting and reposting, publishing advertisements or following applications. As a consequence, these message application users attempt to gather more effective information associated with social attributes from their neighbor nodes so that an accurate message routing-forwarding decision will be achieved. The detailed fuzzy pattern recognition process for node classification is implemented based on three steps, including data quantification, structure construction, and pattern recognition.

\subsubsection{Information Quantification and Determining Membership Degrees for Fuzzy Input}

In OSNs, nodes with multiple different social attributes in unknown online communities try to communicate with each other, so their social attribute characteristics form a strong basis for position locating and data transmission. In general, node profile contains several important social attributes of message application users. To implement a high-performance data transmission process, the FPRDM model reasonably adopts the node profile of message application users to evaluate the special connection relationship between neighbor nodes and destinations.

Specifically, as shown in Figure 1, message application users usually embody multiple different social attributes, such as interests, specialities, physical characteristics, workplaces or residence places. For each attribute of the node $x$, we define a feature vector as a container that is used to load the related and valuable information, and the feature vector is shown as

$$
f v_{\text {node }_{x}}(X)=X_{1}, X_{2}, X_{3}, X_{4} \cdots X_{n}
$$

where $X_{1}, X_{2}, X_{3}, X_{4} \cdots X_{n}$ represent $n$ different social attributes of the message application user node $x_{x}$, and $n$ is the number of social attributes of mobile nodes in the networks. By extension, each of these social attributes can be refined into a smaller eigenvector $e i_{\text {node }}(X)$.

Moreover, when the user node $e_{x}$ contains the same feature value as a destination, the corresponding value of the eigenvector is set to 1 , otherwise to 0 . For example, if the feature vector of interest is

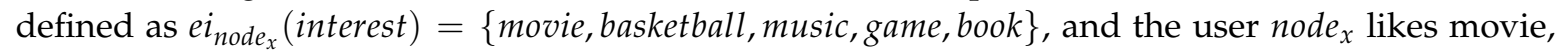
music and book as well as the destinations, then this feature vector could be rigorously initialized to 
$e i_{\text {node }}$ (interest $)=\{1,0,1,0,1\}$. For the sake of the identifiability of the FPRDM system, we set the module length of each feature vector as the fuzzy input of this system, which is obtained by

$$
F I_{\text {node }_{x}}(i)=\left\{\begin{array}{cc}
\left|e i\left(X_{1}\right)\right| & i=1 \\
\left|e i\left(X_{2}\right)\right| & i=2 \\
\left|e i\left(X_{3}\right)\right| & i=3 \\
\cdots & \ldots \\
\left|e i\left(X_{n}\right)\right| & i=n
\end{array}\right.
$$

in which $F I_{\text {node }_{x}}(i)$, namely the quantization vector, denotes different fuzzy inputs of the FPRDM system. Acquired from message application users in the OSNs, the data information associated with social attributes of mobile users is translated from text into a digital format that can be read, recognized, utilized and processed by this system.

Originating from the theory of fuzzy control engineering, the fuzzy inference system is able to transform the piecewise function into some curves, thereby making a vague judgement on the transmission priority of each node in the OSNs. After the process of ambiguity resolution, the specific digital value could be regarded as an accurate assessment foundation for message routing and forwarding decision-making. Refer to the traditional and classical Mamdani fuzzy control system, which is a highly extensible and applicable fuzzy inference model. The FPRDM system model [29] could be established using three continuous components, including Fuzzifier, Fuzzy Inference, and Defuzzifier. Next, a detailed description of the three fuzzy components will be presented in the following sections.

As shown in Figure 1, $F I_{\text {node }_{x}(i)}$, a specified queue defined by Equation (2) is regarded as the fuzzy input of fuzzy pattern recognition and decision-making system, and it is also the discourse domain of variable fuzzy subsets in this system model, further we adopt tripartite method to define three different levels of membership subsets (low, medium and high). Moreover, these related initialization definitions could be formalized as

$$
F S_{\text {node }_{x}}\left(F I_{\text {node }_{x}}(i)\right)=M D_{\text {low }}\left(F I_{\text {node }_{x}}(i)\right), M D_{\text {medium }}\left(F I_{\text {node }_{x}}(i)\right), M D_{\text {high }}\left(F I_{\text {node }_{x}}(i)\right)
$$

where $M D_{\text {low }}\left(F I_{\text {node }_{x}}(i)\right), M D_{\text {medium }}\left(F I_{\text {node }_{x}}(i)\right), M D_{\text {high }}\left(F I_{\text {node }_{x}}(i)\right)$, which respectively correspond to low, medium and high fuzzy subsets, are three different grades of membership degrees for the quantization vector $F I_{\text {node }_{x}}(i)$. As shown in Figure 1, for each message application user node $e_{x}$, this system will generate a corresponding quantization vector. In addition, for each level of membership function, the quantization vector $F I_{\text {node }}(i)$ simultaneously generates $n$ different corresponding membership degrees. The fuzzy subsets $\tilde{A}_{\text {low }}, \tilde{A}_{\text {medium }}$ and $\tilde{A}_{\text {high }}$ are regarded as an evaluation set and the fuzzy inputs are considered as a factor set in the proposed routing strategy, so the mathematical mapping from fuzzy input $F I_{\text {nodex }_{x}}(i)$ to fuzzy subsets could be defined as

$$
\operatorname{MF}\left(F_{\text {nodex }_{x}}(i)\right): R \rightarrow\left\{\tilde{A}_{\text {low }}, \tilde{A}_{\text {medium }}, \tilde{A}_{\text {high }}\right\}
$$

On the basis of the theory of tripartite method, when $\forall e i_{x} \in R$, Equation (4) could be expanded as

$$
M F_{\mu, \eta}\left(F_{\text {node }_{x}}(i)\right)=\left\{\begin{array}{lr}
\tilde{A}_{\text {low }} & F I_{\text {node }_{x}}(i) \leq \mu \\
\tilde{A}_{\text {medium }} & \mu<F I_{\text {node }_{x}}(i)<\eta \\
\tilde{A}_{\text {high }} & F_{\text {node }_{x}}(i)>\eta
\end{array}\right.
$$

where $\mu$ represents a demarcation point between low and medium membership degrees, and $\eta$ is another demarcation point between medium and high membership degrees. Furthermore, $\tilde{A}_{\text {low }}$, $\tilde{A}_{\text {medium }}$ and $\tilde{A}_{\text {high }}$ denote the determined low, medium and high membership function, respectively. 
Accordingly, it is essential for us to define three different levels of membership functions $\tilde{A}_{\text {low }}, \tilde{A}_{\text {medium }}$ and $\tilde{A}_{\text {high }}$ for the quantization vector $F I_{\text {node }_{x}}(i)$ based on the application of the tripartite method, which shows a general mathematical symmetry. In theory, there exist many membership function references in fuzzy control mathematics, such as trapezoidal distribution and semi-trapezoidal distribution, K-order parabolic distribution, Cauchy distribution, normal distribution, and Ridge distribution.

Naturally, in opportunistic social networks, high-tech mobile device users come to generate a large amount of data when accessing the application platform, including social, mobile, behavioral, physical characteristics and a large amount of text information. Generally, when message application users are in urban areas, relatively developed communication infrastructures contribute to a high-speed and stable network environment. Therefore, the frequent communication among mobile nodes in an online community is likely to generate a great amount of social information. On the contrary, poor and dated communication infrastructures in suburbs or remote areas may result in congested, slow and intermittent communications between mobile users [1], hence there will be less social information about mobile users in this type of network environment. Furthermore, hot issues, novel topics, popular entertainment, and public preferences are the areas that the public is deeply concerned about, even though the distinction in age, occupation, and gender may lead to a slight difference in attention. Through strict data analysis and theoretical proof, the data related to social attributes that come from message application users should be subject to a relatively standard normal distribution [2].

Consequently, through referring to the normal distribution membership functions in a traditional fuzzy control system and the existing data distribution model in opportunistic mobile social networks, $\tilde{A}_{\text {low }}, \tilde{A}_{\text {medium }}$ and $\tilde{A}_{\text {high }}$ could be strictly calculated by

$$
\begin{aligned}
& F_{\tilde{A}_{\text {low }}}\left(F I_{\text {node }_{x}}(i)\right)= \begin{cases}1 & F I_{\text {node }_{x}}(i) \leq a_{1} \\
e^{-\left(\frac{F I_{\text {node }_{x}(i)}-a_{1}{ }^{2}}{\sigma_{1}}\right)} & F I_{\text {node }_{x}}(i)>a_{1}\end{cases} \\
& F_{\tilde{A}_{\text {medium }}}\left(F I_{\text {node }_{x}}(i)\right)=e^{-\left(\frac{F I_{\text {node }_{x}(i)}-a_{2}}{\sigma_{2}}\right)^{2}} \\
& F_{\tilde{A}_{\text {high }}}\left(F I_{\text {node }_{x}}(i)\right)= \begin{cases}0 & F I_{\text {node }_{x}}(i) \leq a_{3} \\
e^{-\left(\frac{F I_{\text {node }_{x}(i)}-a_{3}}{\sigma_{3}}\right)^{2}} & F I_{\text {node }_{x}}(i)>a_{3}\end{cases}
\end{aligned}
$$

in which $a_{1}, a_{2}$ and $a_{3}$ represent three different levels of location parameters, and $\sigma_{1}, \sigma_{2}$ and $\sigma_{3}$ denote the scale parameter for the low, medium and high membership functions $F_{\tilde{A}_{\text {low }}}, F_{\tilde{A}_{\text {medium }}}$ and $F_{\tilde{A}_{\text {high }}}$, respectively. As exhibited in Figure 2a-c, when the quantization vector changes over time, low, medium and high membership functions present different curves and the codomain is always between 0 and 1. Eventually, the trend of these three membership functions depends on the location parameter and scale parameter, which could be determined by the distribution of the data obtained from message application users. 


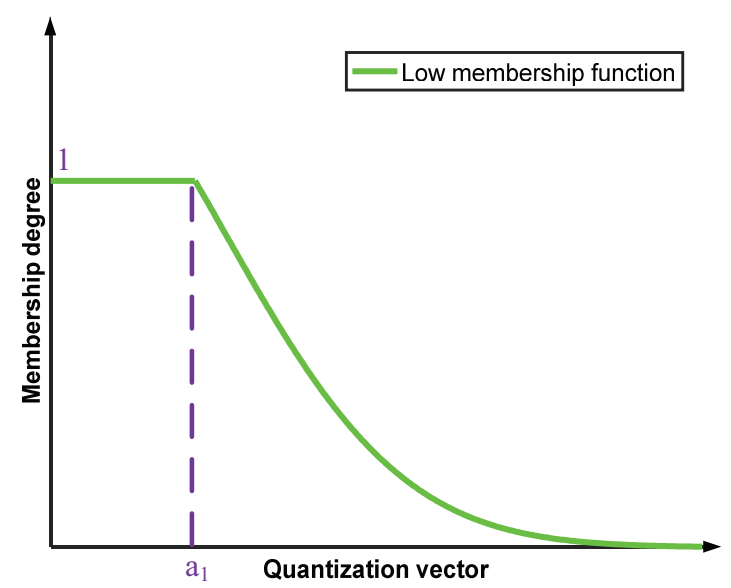

(a)

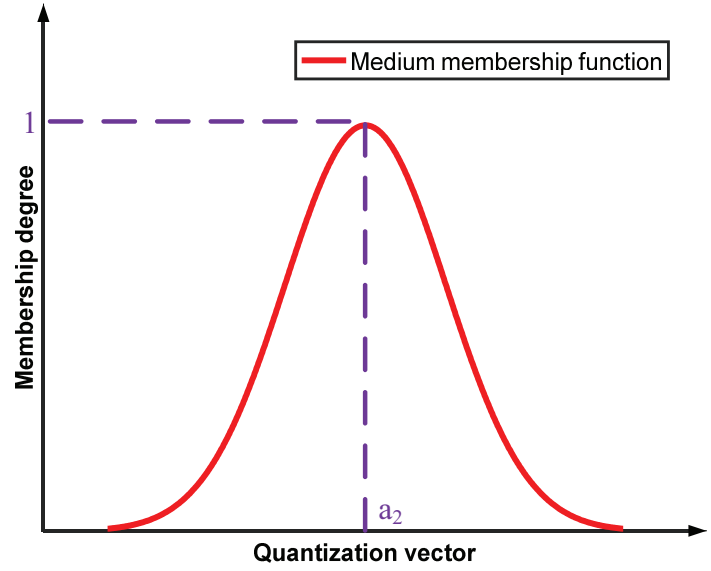

(b)

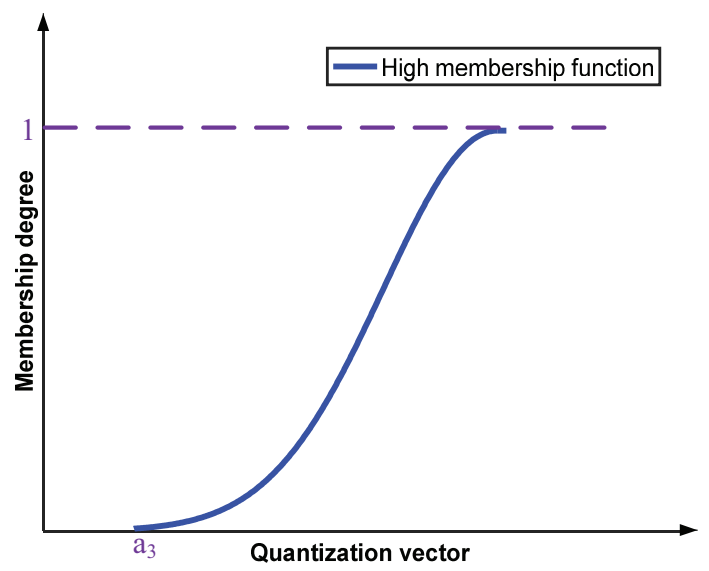

(c)

Figure 2. Three different grades of membership functions quantization vectors.

\subsubsection{Fuzzy Pattern Recognition for Node Classification in Opportunistic Mobile Social Networks}

In opportunistic mobile social networks, two message application users, which possess a relatively high social similarity between them, are more likely to be in the same online community, and information communication between them is expected to be more frequent. Therefore, a user with a high social similarity to the destination should be a reliable relay node in the data transmission process. Then, the process of pattern recognition for node classification employs fuzzy inference logic to evaluate the social similarity between mobile users, so as to select some suitable relay nodes for message routing and forwarding, and eventually achieves an efficient and sustainable data transmission link.

For effectively assessing the pattern recognition results of node classification, we define the membership degree of each quantization vector as $\underline{A}_{m n}$, in which $m$ represents different levels of membership subsets and $n$ denotes different fuzzy input values. Thereupon, each fuzzy subset could be represented by $n$ different fuzzy inputs, which is mathematically quantified as

$$
\begin{gathered}
\underline{A}_{m}=\left\{\begin{aligned}
\tilde{A}_{\text {low }} & m=1 \\
\tilde{A}_{\text {medium }} & m=2 \\
\tilde{A}_{\text {high }} & m=3
\end{aligned}\right. \\
\underline{A}_{m}\left(F I_{\text {node }_{x}}(i)\right)=\left(\underline{A}_{m 1}\left(F I_{\text {node }_{x}}(i)\right), \underline{A}_{m 2}\left(F I_{\text {node }_{x}}(i)\right) \cdots \underline{A}_{m n}\left(F I_{\text {node }_{x}}(i)\right)\right) \quad(m=1,2,3)
\end{gathered}
$$


where $\underline{A}_{m n}\left(F I_{\text {node }_{x}}(i)\right)$ indicates the membership degree of $F I_{\text {node }_{x}}(i)$ from the membership subset $M D_{m}$. In a traditional fuzzy inference system, the goal of the defuzzifier component is to convert fuzzy membership subsets and membership degrees into an accurate judgment value. As a commonly-used approach to calculate the membership degree in a traditional fuzzy inference system [6], maximum membership principle means the membership degree of each level (low, medium, and high) is equal to the maximum membership of $n$ quantization vectors from the corresponding level. Consequently, the membership degree $\underline{A}_{m}\left(F I_{\text {node }}(i)\right)$ of fuzzy input $F I_{\text {node }}(i)$ for membership subset $M D_{m}\left(F I_{\text {node }_{x}}(i)\right)$ could be calculated by

$$
\underline{A}_{m}\left(F I_{\text {node }_{x}}(i)\right)=\bigwedge_{i=1}^{n}\left\{\underline{A}_{m i}\left(F I_{\text {node }_{x}}(i)\right)\right\}(m=1,2,3)
$$

Generally, there are several paradigms of defuzzification strategies in a fuzzy inference system, such as center of area (CoA) or mean of maximum (MoM) [29]. The maximum membership principle will be regarded as the defuzzification method in the proposed algorithm due to its expansibility. Based on that, the membership degree of fuzzy control result $D_{\text {node }}$ for pattern recognition of node classification could be expressed as the union of three different membership degrees that correspond to three different levels of membership subsets, which is shown as

$$
\begin{aligned}
D_{\text {node }_{x}} & =\bigvee_{m=1}^{3}\left\{\bigwedge_{i=1}^{n}\left\{\underline{A}_{m i}\left(F I_{\text {node }_{x}}(i)\right)\right\}\right\} \\
& =\underline{A}_{\text {low }}\left(F I_{\text {node }_{x}}(i)\right) \bigvee \underline{A}_{\text {medium }}\left(F I_{\text {node }_{x}}(i)\right) \bigvee \underline{A}_{\text {high }}\left(F I_{\text {node }_{x}}(i)\right)
\end{aligned}
$$

After determining the threshold $K$ accurately, we can compare $D_{\text {node }_{x}}$ with $K$ to evaluate whether the node $_{x}$ is a suitable relay node. To be specific, when the value of $D_{\text {node }_{x}}$ is greater than or equal to $K$, the $\operatorname{node}_{x}$ may be an appropriate next hop; otherwise, the node may not be suitable to participate in the process of message routing and forwarding.

\subsection{Reasonable Weight Allocation and Inference of Fuzzy Relationships Via the Analytic Hierarchy Process}

The main aim of fuzzy pattern recognition is to present a preliminary judgment on node classification. For the sake of a more precise decision-making on data transmission, it is essential to execute the process of weight adjustment and fuzzy relationship inference. By synthetically evaluating the control result of pattern recognition and fuzzy relation inference, message application users are able to determine the next hop, even the third hop, from their neighbor nodes efficiently.

The procedure of weight adjustment plays a significant role in fuzzy relation evaluation, which aims to reasonably assess the status of each fuzzy input in the whole data transmission. The social attributes of nodes are layered and interlaced, which is specifically reflected in the fact that the social attributes of message application users are related to each other. Therefore, we adopt the analytic hierarchy process (AHP) [30] to evaluate the weight of their social attributes in routing selection and data forwarding.

Firstly, we need to determine the comparison weight between $n$ different fuzzy inputs and construct a $n$-order comparison matrix, which is shown as Table 1.

Table 1. Weight scale for $n$ different fuzzy inputs.

\begin{tabular}{ccccc}
\hline $\mathbf{W}$ & $\left|e i\left(X_{\mathbf{1}}\right)\right|$ & $\left|e i\left(X_{\mathbf{2}}\right)\right|$ & $\cdots$ & $\left|e i\left(X_{n}\right)\right|$ \\
\hline$\left|e i\left(X_{1}\right)\right|$ & 1 & $R a_{(1,2)}$ & $\cdots$ & $R a_{(1, n)}$ \\
$\left|e i\left(X_{2}\right)\right|$ & $1 / R a_{(1,2)}$ & 1 & $\cdots$ & $R a_{(2, n)}$ \\
$\vdots$ & $\vdots$ & $\vdots$ & $\ddots$ & $\vdots$ \\
$\left|e i\left(X_{n}\right)\right|$ & $1 / R a_{(1, n)}$ & $1 / R a_{(2, n)}$ & $\cdots$ & 1 \\
\hline
\end{tabular}


AHP directly and effectively combines the results of human experience and objective judgment of machines, and quantitatively describes the importance of pairwise comparison of elements in a hierarchy. Then, the weight value reflecting the relative importance order of elements at each level is calculated by mathematical method, and the relative weight of all elements is calculated and sorted by the total order between all levels [30]. The adjustment of weight parameters in our experiment is based on the characteristics of the real data set. Consequently, from Table 1 , the weight ratio $R a(i, j)$ is the ratio of all the elements in the first row to all the elements in the first column, which could be set as

$$
R a_{(i, j)}=\frac{\left|e i\left(X_{i}\right)\right|}{\left|e i\left(X_{j}\right)\right|}
$$

Then, we normalize each of the columns in the above matrix $C M$, which can be shown as

$$
R b_{(i, j)}=\frac{R a_{(i, j)}}{\sum_{i=1}^{n} R a_{(i, j)}}
$$

where $\sum R a(i, j)$ denotes the sum of the $R a(i, j)$ for each column and $R b(i, j)$ is the new comparison weight. After that, the eigenvector $w_{i}$ of the $i$-th fuzzy input is summed from each row of the new matrix, which can be formalized as

$$
\begin{aligned}
B_{(i)} & =\sum_{j=1}^{n} R b_{(i, j)} \\
w_{i} & =\frac{B_{(i)}}{\sum_{i=1}^{n} B_{(i)}} .
\end{aligned}
$$

To reasonably record the weight value of each social attribute of mobile nodes in the networks, the proposed algorithm loads the weight value corresponding to each fuzzy input into a row vector. Then, the weight vector $W_{(} w_{1}, w_{2} \cdots w_{n)}$ for $n$ different fuzzy inputs could be defined as

$$
\left.W_{(} w_{1}, w_{2} \cdots w_{n}\right)=\left[w_{1}, w_{2}, w_{3} \cdots w_{n}\right]
$$

Based on the above formulas, $n$ weight values for $n$ different fuzzy inputs (social attributes of nodes) can be obtained from $w_{i}$. After determining the importance of each social attribute of message application users on the process of message routing and forwarding, the system comes to assign each fuzzy input an appropriate weight automatically, which is in favor of the final comprehensive evaluation of the fuzzy relationship between fuzzy input factor sets and fuzzy subsets. Additionally, the fuzzy relationship matrix $R_{f}$ could be induced by $f$, which can be represented as

$$
R_{f}=\left[\begin{array}{ccc}
\underline{A}_{11}\left(F I_{\text {node }_{x}}(1)\right) & \underline{A}_{21}\left(F I_{\text {node }_{x}}(1)\right) & \underline{A}_{31}\left(F I_{\text {node }_{x}}(1)\right) \\
\underline{A}_{12}\left(F I_{\text {node }_{x}}(2)\right) & \underline{A}_{22}\left(F I_{\text {node }_{x}}(2)\right) & \underline{A}_{32}\left(F I_{\text {node }_{x}}(2)\right) \\
\underline{A}_{13}\left(F I_{\text {node }_{x}}(3)\right) & \underline{A}_{23}\left(F I_{\text {node }_{x}}(3)\right) & \underline{A}_{33}\left(F I_{\text {node }_{x}}(3)\right) \\
\vdots & \vdots & \vdots \\
\underline{A}_{1 n}\left(F I_{\text {node }_{x}}(n)\right) & \underline{A}_{2 n}\left(F I_{\text {node }_{x}}(n)\right) & \underline{A}_{3 n}\left(F I_{\text {node }_{x}}(n)\right)
\end{array}\right]
$$

where $\underline{A}_{1 i}\left(F I_{\text {node }_{x}}(i)\right), \underline{A}_{2 i}\left(F I_{\text {node }_{x}}(i)\right)$ and $\underline{A}_{3 i}\left(F I_{\text {node }_{x}}(i)\right)$ denote low, medium and high membership degrees of the $i$-th fuzzy input of the message application user node $e_{x}$, respectively. Through synthetically considering the impact of different weights of fuzzy inputs, the fuzzy transformation mapping $B_{n o d e_{x}}$ from $U_{\text {node }}^{\prime}$ to $V_{n o d e_{x}}$ is calculated by

$$
\begin{aligned}
B_{\text {node }_{x}} & \left.=W_{(} w_{1}, w_{2} \cdots w_{n}\right) \circ R_{f} \\
& =\underline{b}_{\text {node }_{x}}(1), \underline{b}_{\text {node }_{x}}(2) \cdots \underline{b}_{\text {node }_{x}}(n) .
\end{aligned}
$$


After assessing the importance of each social attribute of message application users, the FPRDM system comes to assign a high weight value to the attribute feature that performs a relatively vital role in the data transmission process. And on the contrary, a small weight value will be allocated to other general fuzzy inputs. Additionally, the fuzzy mapping from factor set to evaluation set actually is to explore the fuzzy link between social attributes and message routing-forwarding. Hence the value of $B_{\text {node }_{x}}$ could be used as an important basis for fuzzy decision after defuzzification.

\subsection{Information Fusion and Fuzzy Decision-Making for Message Routing-Forwarding in Opportunistic Mobile Social Networks}

On the grounds of the constant and slight change of attribute features of message users in the data transmission process, it must be inaccurate to evaluate the transmission priority of nodes through some exact values of these attribute features at a certain time. This also leads to a large deviation in decision-making on node classification and data transmission. Therefore, through analysing the network routing environments and the distribution of the data related with node movement, the fuzzy inference system can be employed to comprehensively evaluate the transmission relationship between relay nodes and destinations, constructing a relatively complete and efficient message routing-forwarding link.

To effectively quantify the whole process of data transmission from source nodes to destinations, this model defines $C R_{\left(\text {node }_{x}\right)}$ and $I R_{\left(\text {node }_{x}\right)}$ as the final fuzzy control result and the inference result of fuzzy relationship for $n o d e_{x}$, respectively, and the two definitions are shown as

$$
\left\{\begin{aligned}
C R_{\text {node }_{x}} & =c_{1}, c_{2}, c_{3}, c_{4} \cdots c_{q} \\
I R_{\text {node }_{x}} & =r_{1}, r_{2}, r_{3}, r_{4} \cdots r_{e}
\end{aligned}\right.
$$

where $c_{1}, c_{2}, c_{3}, c_{4} \cdots c_{q}$ represent different fuzzy subsets that the node $e_{x}$ belongs in, and $r_{1}, r_{2}, r_{3}, r_{4} \cdots r_{e}$ denote $e$ different fuzzy transformation relationships from factor set $U_{\text {node }_{x}}$ to evaluation set $V_{\text {node }_{x}}$.

For more accurately assessing the transmission relationship between nodes, feature vectors $C R_{\text {node }_{x}}$ and $I R_{\text {node }_{x}}$ will be reasonably digitized to specific values $D_{\text {node }_{x}}$ and $B_{\text {node }_{x}}$, respectively. Eventually, the above system of equations (20) could be translated into another form, which is shown as

$$
F_{\text {node }_{y}}=F R D M\left(D_{\text {node }_{x}}, B_{\text {node }_{x}}\right)
$$

where FRDM denotes the process of fuzzy recognition and decision-making for node classification and data transmission, and $D_{n o d e_{x}}$ and $B_{\text {node }}$ represent the membership degree of fuzzy control and fuzzy relationship inference, respectively. Since the fluctuation ranges of $D_{\text {node }}$ and $B_{\text {node }}$ are both from 0 to 1, the FPRDM strategy adopts the tanh function (shown as Equation (22)) to comprehensively evaluate the transmission value $T V$, which can be regarded as the final reference to data transmission between relay nodes and destinations.

$$
\begin{aligned}
T V & =\tanh \left(D_{\text {node }_{x}}+B_{\text {node }_{x}}\right) \\
& =\frac{e^{\left(D_{\text {nodex }}+B_{\text {nodex }}\right)}-e^{-\left(D_{\text {nodex }}+B_{\text {nodex }}\right)}}{e^{\left(D_{\text {nodex }}+B_{\text {nodex }}\right)}+e^{-\left(D_{\text {nodex }}+B_{\text {nodex }}\right)}} .
\end{aligned}
$$

From the above formula, when $\left(D_{\text {node }_{x}}+B_{\text {node }_{x}}\right)$ is between 0 and 2, the slope of $F_{\text {node }_{y}}$ is relatively large and the value of $T V$ fluctuates enormously, which is conducive to clearly distinguish the transmission priority between nodes. On the basis of feedback mechanism, message application users are able to make an accurate decision on message routing and forwarding by comparing the $T V$ value of the future two-hop nodes during the data transmission process.

Theoretically, most of the traditional and classical routing strategies in opportunistic mobile social networks commonly seeks the next-hop node only from its neighbors and then forward data packets, which is likely to cause the interruption or instability of data transmission. 
Consequently, we investigate a routing feedback mechanism approach, concentrating on not only the next-hop nodes but also the third-hop nodes, which facilitates the construction of a continuous and stable link in the data transmission process.

To be specific, as shown in Figure $3, n^{2} e_{A}, n$ ode $e_{B}$ and node $C$ are in the online community 1 at the current moment. Additionally, node $e_{D}$ and node $_{E}$ are in the online community 2, while node ${ }_{F}$, node $_{G}$, node $_{H}$ and node $e_{I}$ are in the online community 3. node $A$ and node $e_{G}$ are respectively the source node and destination. Moreover, the black point represents the relay node, while the red one denotes an irrelevant node in opportunistic social networks. The number of mobile nodes in a community represents the corresponding node density. Each message application user calculates its own TV according to the attribute characteristics of the destinations provided by the source node $A$. As a message carrier, node ${ }_{A}$ comes to collect and compare the $T V$ values of its future two-hop message users. As shown in Figure 3, because $T V_{I}>T V_{E}$ and $T V_{E}>T V_{C}$, the source node node $A$ only transmits data packets to node $_{C}$, and then sends data packets to node $e_{E}$ and node $e_{I}$. Finally, these data packets will be forwarded to the destination $n_{\text {ode }}$. According to the different node density and membership degree within each online community, the proposed scheme comes to adopt different strategies to implement message routing and forwarding. The duplicate controlling model is adopted among the mobile nodes in the same community. Moreover, due to a low node density within the online community 2 , node $\mathrm{C}$ transmits messages to $n_{0} e_{E}$ via the mechanism of copying messages, which is able to avoid the loss of data packets. On the contrary, the node density in community 3 is relatively high, hence $n o d e_{E}$ employs the method of direct forwarding to transmit messages to node ${ }_{I}$, and more importantly, preventing the flooding of data packets in the online community.

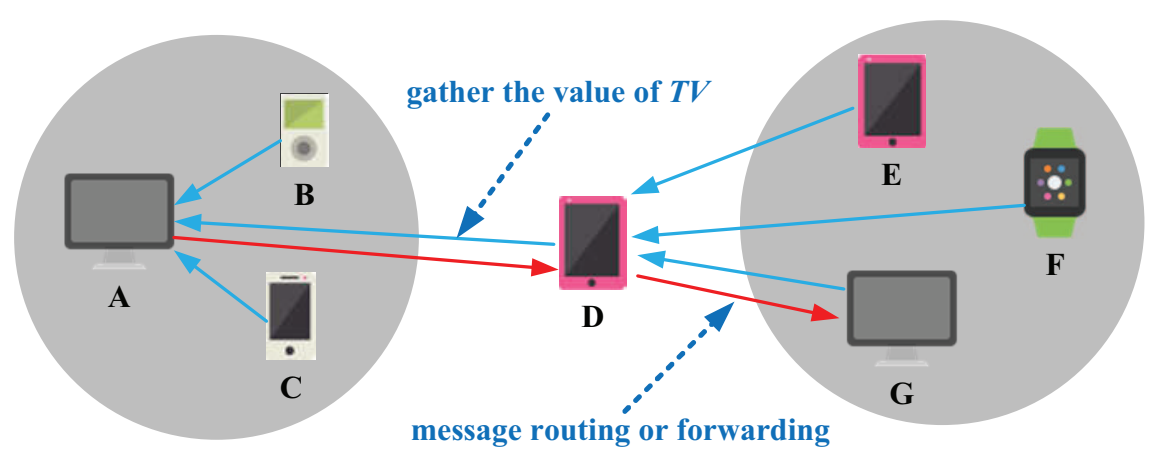

Online community 1

Online community 2

Figure 3. The fuzzy decision-making process for data transmission in opportunistic mobile social networks.

\subsection{Algorithm Complexity Analysis}

To describe the extensibility and applicability of fuzzy recognition and the decision-making system, we rigorously built the Fuzzy pattern cognition and decision model (shown as Algorithm 1) to show the time complexity of this system. Specifically speaking, in the process of determining the membership degree of each message user, $n$ attribute features may correspond to three levels of fuzzy subsets (low, medium and high) that contain $3 n$ membership degrees values, which presents the time complexity of $O(3 n)$. Additionally, the time complexity of the analytic hierarchy process [30] is $O(n)$, because there exist $n$ different attribute features which need to be assigned $n$ different weight values. Ultimately, in the process of fuzzy decision-making for message routing and forwarding, source nodes and message carriers transmit data packages to their future two-hop nodes by gathering and comparing the value of $T V$, therefore the corresponding time complexity is $O\left(\log _{2} n\right)$. Consequently, the total time complexity of fuzzy recognition and decision-making models for opportunistic social networks can be calculated by $O\left(3 n+n+\log _{2} n\right)=O(n)$, which highlights a relatively low calculation consumption of this model. 


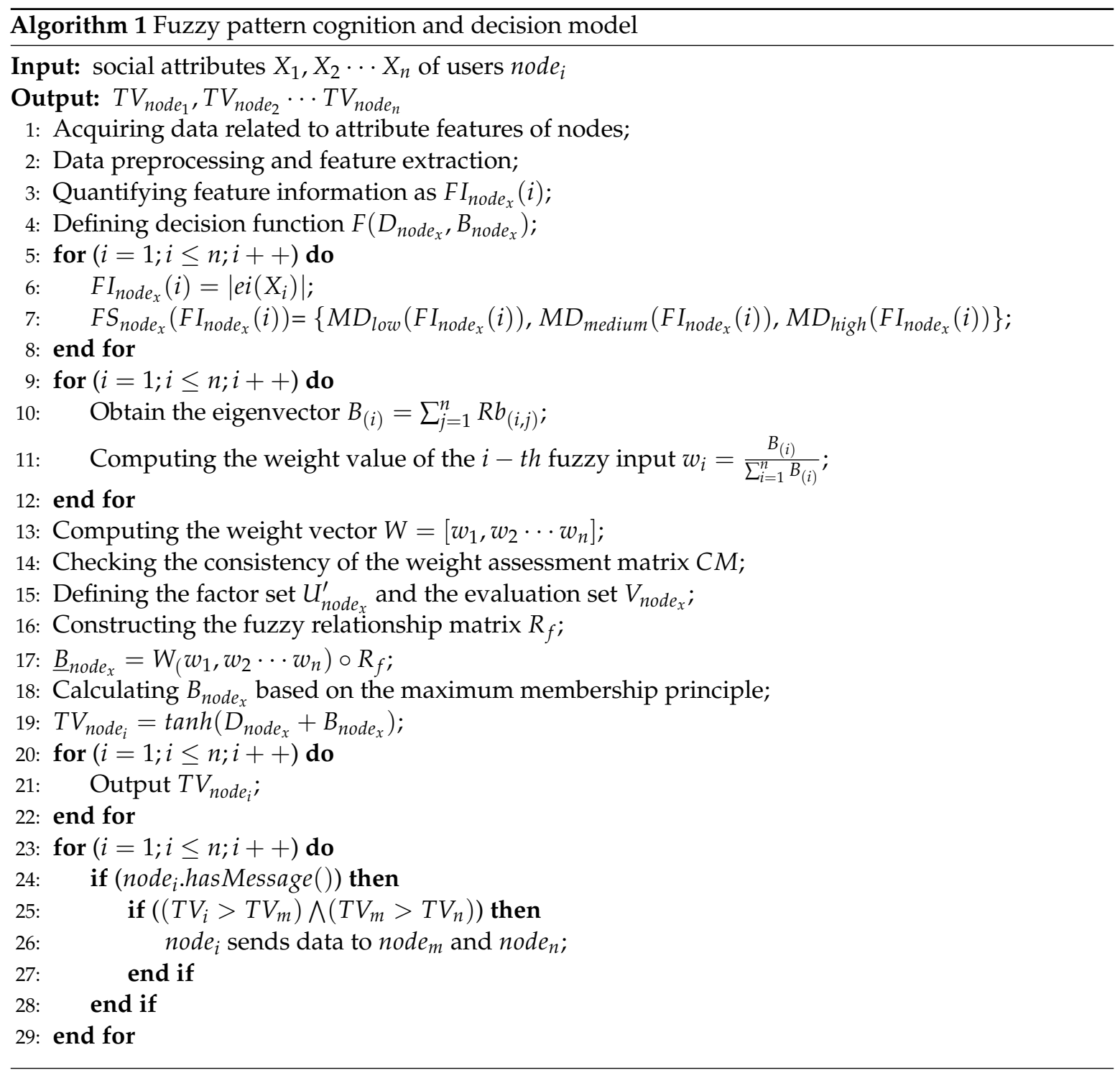

\section{Simulation And Analysis}

In our experiment, the simulator opportunistic network environment (ONE) is utilized to evaluate the experimental performance of the FPRDM routing algorithm, and the mathematical tool MATLAB could be used to assign different weight values to each social features of mobile users in OSNs. In addition, to comprehensively assess the performance of the FPRDM routing algorithm, it will be compared with other four traditional or latest routing protocols in OSNs: Spray and wait [13], Epidemic [2], ICMT (information cache management and data transmission algorithm exploiting comprehensive node similarity) [9], and FCNS (a fuzzy routing-forwarding algorithm exploiting comprehensive node similarity) [6]. Spray and wait, and Epidemic are traditional methods in opportunistic social networks. ICMT attempts to achieve an efficient routing connectivity using memory management and node collaboration, while the FCNS is a fuzzy routing-forwarding algorithm based on the Mamdani fuzzy system.

\subsection{Setting of Experimental Parameters}

In this experiment, for constructing a simulation environment which is conducive to testing the performance of FPRDM algorithm, this paper employs the real datasets acquired from the Community Resource for Archiving Wireless Data At Dartmouth (CRAWDAD), which consists of data sets Infocom 
5, Infocom 6, Cambridge and Intel (Table 2), and the experimental setting of parameters is shown as Table 3.

Table 2. The mobility model of the simulation environment.

\begin{tabular}{ccccc}
\hline Dataset & Infocom5 & Infocom6 & Cambridge & Intel \\
\hline Device & iMote & iMote & iMote & iMote \\
Duration (days) & 3.5 & 4 & 11 & 3.5 \\
Number of experimental devices & 41 & 98 & 50 & 8 \\
Number of internal contacts iMote & 2245 & 1706 & 1087 & 1364 \\
\hline
\end{tabular}

Table 3. The experimental setting of the simulation environment.

\begin{tabular}{cc}
\hline Simulation Environment & Description \\
\hline Simulator & Opportunistic Network Environment (ONE) \\
Communication area & $3000 \times 3000$ \\
Total simulation time $(\mathrm{h})$ & $10-20$ \\
Number of nodes $\mathrm{N}$ & 100 (initial value), 200, 400, 600 \\
Cache space of a node $C(\mathrm{Mb})$ & 10 (initial value), $15,20,25,30,35,40$ \\
Speed of a node $(\mathrm{m} / \mathrm{s})$ & $1-25$ \\
Initial energy for a node $(\mathrm{J})$ & 200 \\
Number of social features & 8 \\
Three location parameters $a_{1}, a_{2}, a_{3}$ & $a_{1}=0.75, a_{2}=0.5, a_{3}=0.25$ \\
Three scale parameters $\sigma_{1}^{2}, \sigma_{2}^{2}, \sigma_{3}^{2}$ & $\sigma_{1}^{2}=\sigma_{2}^{2}=\sigma_{3}^{2}=0.96$ \\
\hline
\end{tabular}

In particular, the movement trajectory of mobile users in the communication area is subject to the datasets in Table 2 [42], because these models could be based on community division and node profiles. Furthermore, the total energy for a node is initialized to $200 \mathrm{~J}$, and each data transmission consumes $0.25 \mathrm{~J}$ of energy. The speed for a node fluctuates from 1 to $25 \mathrm{~m} / \mathrm{s}$, which could be the mobile speed of walking, biking, or driving. As shown in Figure 4, for each type of the real data set and each group of parameter setting, a set of simulation data is generated in this experiment, therefore 24 different sets of data will be obtained from 24 simulation experiments. Because three measurement metrics in this experiment cannot reach the highest value at the same time, the delivery ration is given priority in the optimization process. Among the 24 groups of experimental data, the parameters are generally set based on the optimal group of experimental data (the highest delivery ration and relatively low delay), which is referred to the optimal performance in the 10th experiment from Figure 4 . Referring to the parameter initialization from the paradigm of fuzzy inference system and experimental results, the location and scale parameters are set as $a_{1}=0.75, a_{2}=0.5, a_{3}=0.25$, and $\sigma_{1}^{2}=\sigma_{2}^{2}=\sigma_{3}^{2}=0.96$ from the parameter settings of the 10th experiment. In addition, based on the common settings of the opportunistic network environment, the number of nodes in this communication area $N$ is set as 100, 200, 400 and 600, and the cache space for a node $C$ is set as 10, 15, 20, 25, 30, 35, and $40 \mathrm{MB}$. 


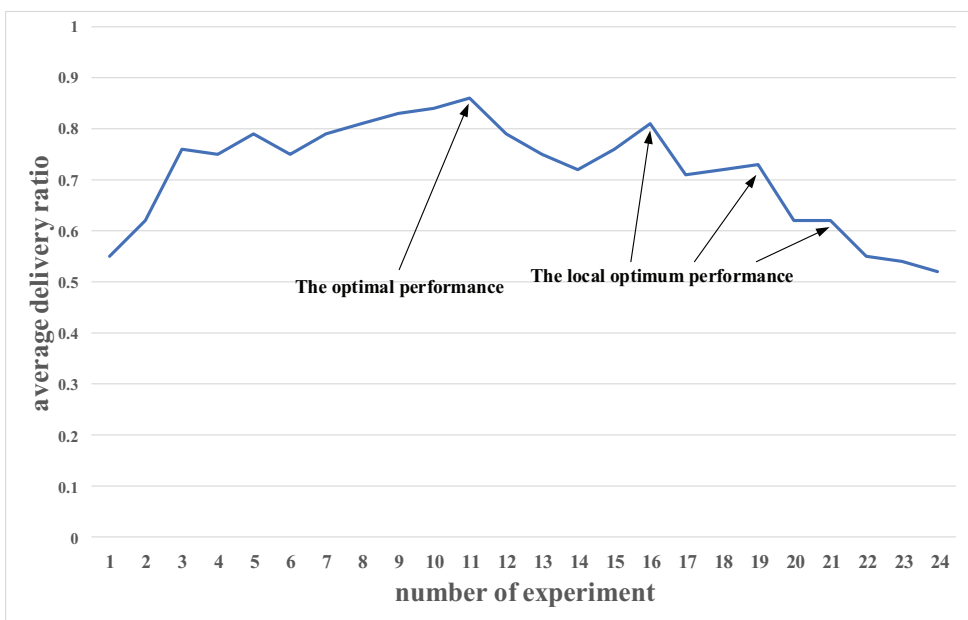

Figure 4. The average delivery ratio from each group of experimental data.

Moreover, because the social attribute information of nodes does not exist in the four real data sets, this simulation defines eight different social characteristics for each node, including interests, occupation, common friends, preferences, browsing history, repost record, comments, and online time. Each of these social features consists of several feature words, and each mobile user randomly corresponds to a different number of feature words. This simulation allocates specific feature words to each mobile node in the communication area. If a pair of nodes have more identical feature words, the opportunities of message transmission between them are even greater. Moreover, the number of repeated feature words also reflects the weight of the corresponding social attribute. If a social attribute includes more repeated feature words, then this attribute can be considered as a more important foundation to find the destination node. As shown in Figure 5, the number of repeated feature words in social attributes "preference", "browsing history", and "comment" are relatively less, while the social attributes of "interest", "occupation", and "common friend" present more identical feature words in the simulation. As shown in Figure 6, the analytic hierarchy process (AHP) algorithm allocates weights to each social characteristic based on the four real data sets. This also indicates that the social features of "interest", "occupation", and "common friend" play a relatively important role in the data transmission process, while the characteristics of "preference", "browsing history", and "comment" are not particularly important on community division.

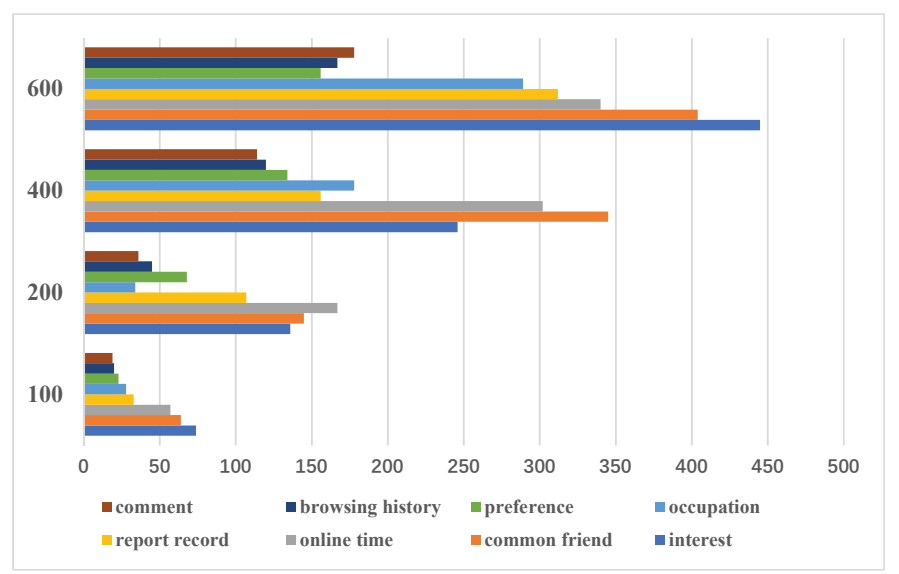

Figure 5. The number of repeated feature words for mobile nodes in the communication domain. 


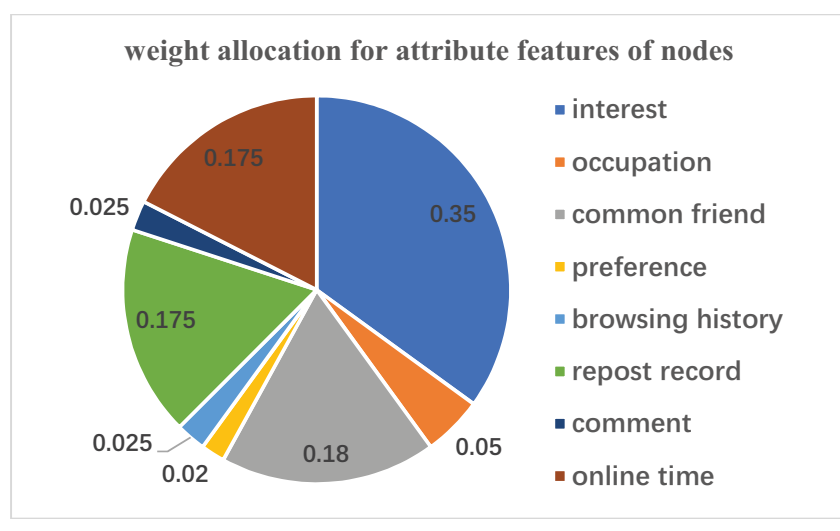

Figure 6. Weight value distribution for social features of mobile users.

\subsection{Experimental Measurement Metrics}

For evaluating the performance of the FPRDM routing algorithm comprehensively, the final result obtained from this experiment is the average value of the four testing results from the above mobility models, and we will analyze the experimental results in terms:

(1) Delivery ratio: This measurement metric represents the probability of neighbor and destination nodes acquiring data packets, which is expressed as

$$
D_{\text {node }}=D_{\text {receive }} / D_{\text {send }}
$$

where $D_{\text {receive }}$ represents the number of messages obtained from the neighbor nodes in the same online community, and $D_{\text {send }}$ denotes the number of messages transmitted by mobile users. There may be data packets loss and packet damage phenomenon in the data dissemination process, so $D_{\text {send }}>D_{\text {receive }}$ and $D_{\text {node }}<1$.

(2) Average end-to-end delay: This parameter is the average delay of routing selection, waiting for messages, node mobility, and message forwarding. It could be computed as

$$
A_{\text {node }}=A_{\text {sum }} / \mathrm{N}
$$

in which $A_{\text {sum }}$ denotes the total delay of data transmission from source nodes to destinations and $N$ represents the total number of mobile users in the communication area.

(3) Network overhead on average: This parameter denotes the average overhead of a successful message forwarding between a pair of mobile users. The network overhead on average is the network overhead of each mobile node in data transmission, including memory consumption, time delay between nodes, energy consumption, etc. Therefore this parameter can be expressed as the ratio of total network overhead to the number of nodes in the communication area, which could be formalized as

$$
O_{\text {node }}=O_{f o r}-O_{s e c} / N
$$

where $O_{f o r}$ is the total time of data transmission and $O_{s e c}$ represents the total time of successful message forwarding between mobile users, and $N$ denotes the total number of mobile nodes.

\subsection{Analysis of Experimental Results}

In this experiment, we mainly pay attention to the impact of the cache space and number of nodes on the performance of various routing algorithms. In big data and $5 \mathrm{G}$ network environments, due to the large amount of transmitted data between mobile devices, the cache space of a node becomes a crucial index to measure the performance of routing strategies in OSNs. In addition, defined by the number of nodes and mobile pattern, the network topology has also been an essential consideration in the data transmission process. 
Firstly, we investigate the impact of the cache space and number of mobile devices on delivery ratio. As shown in Figure 7, with the increase of the cache space and number of nodes, the delivery ratio from these five routing algorithms generally maintains a slow upward trend, and FPRDM scheme shows a slight enhancement as compared with other four routing protocols. In particular, from Figure $7 \mathrm{~d}$, when $N=600$ and $C=40 \mathrm{Mb}$, the delivery ratio from FPRDM is about 0.88 , which is the highest value this algorithm shows. Moreover, from Figure $7 \mathrm{a}$, when $N=100$ and $C=25 \mathrm{Mb}$, the delivery ratio from the FCNS routing protocol exceeds that from FPRDM for only the one time.

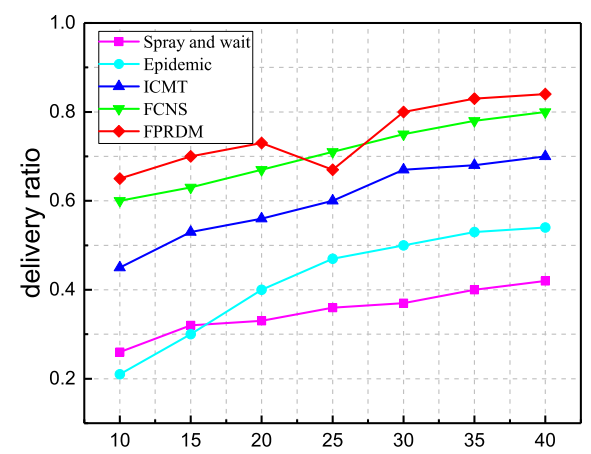

(a) Cache (Mb)

(a)

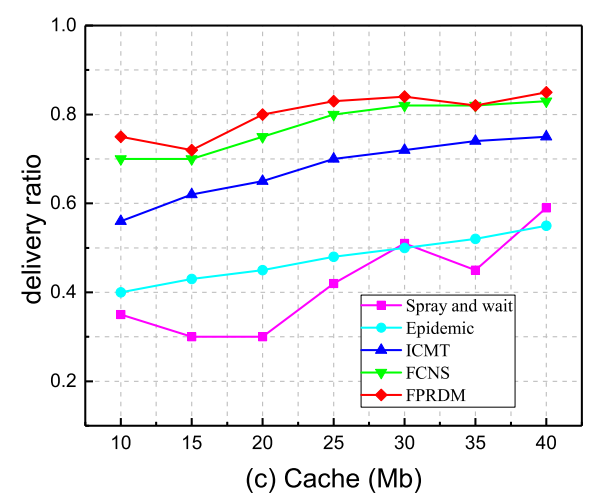

(c)

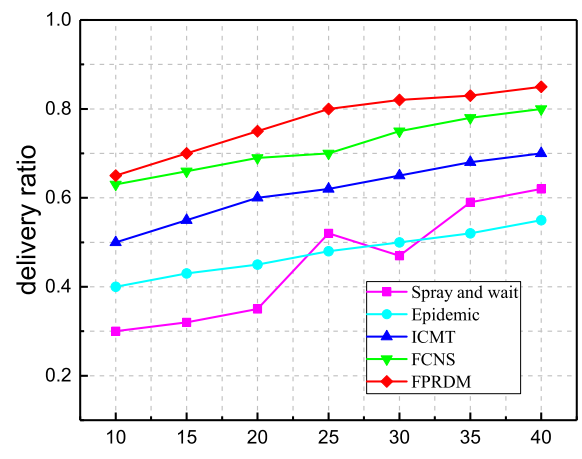

(b) Cache (Mb)

(b)

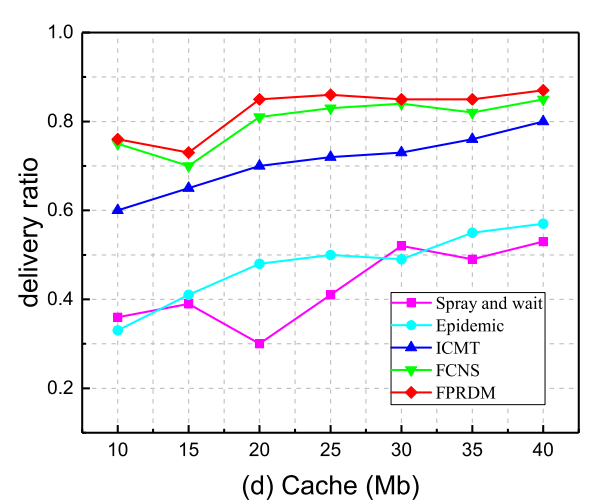

(d)

Figure 7. Performance comparison of delivery ratio with various cache spaces and various number of nodes. (a) $N=100$. (b) $N=200$. (c) $N=400$. (d) $N=600$.

As shown in Figure 7, the delivery ratio of the ICMT routing algorithm is always in the middle degree, while the traditional routing approaches Epidemic and Spray and Wait present a relatively low delivery ratio all the time. This is because these methods only roughly control the number of message replications or data packages, but do not pay attention to the life cycle time of the message or changes in network topology. When selecting a suitable relay node, the Epidemic routing algorithm adopts the flooding model to widely spread data packages in the network, so the large number of message duplicates circulating in the networks may promote the increase of delivery ratio, and cause a sharp rise in costs and delays. ICMT, Spray and Wait, and FCNS employ data management, encountering history, and fuzzy control to make a more accurate decision on message routing and forwarding, respectively. However, these approaches still transmit messages to multiple unrelated nodes which do not participate in the data dissemination process. The FPRDM scheme first performs a classification identification of nodes, and then allocates a suitable weight to each social characteristic of message user and determines three different grades of membership functions (high, medium, and low) 
for the quantization vector, thereby selecting the appropriate relay nodes through the process of a defuzzifier component. For this reason, a relatively high delivery ratio with an average of 0.8 could be presented from the FPRDM scheme.

Furthermore, Figure 8 displays the performance comparison of these five routing algorithms in terms of the average end-to-end delay with various cache spaces and various number of nodes. On the whole, the Epidemic routing algorithm consistently exhibits the highest end-to-end delay among these strategies due to the flooding dissemination of messages. Additionally, because the Spray and Wait and ICMT routing protocols respectively employ two-phases and a cooperative mechanism to reduce the number of messages appropriately, they present an average end-to-end delay of $300 \mathrm{~s}$ when enlarging the cache space of mobile devices from 10 to $40 \mathrm{Mb}$, which is within the middle range among these approaches.

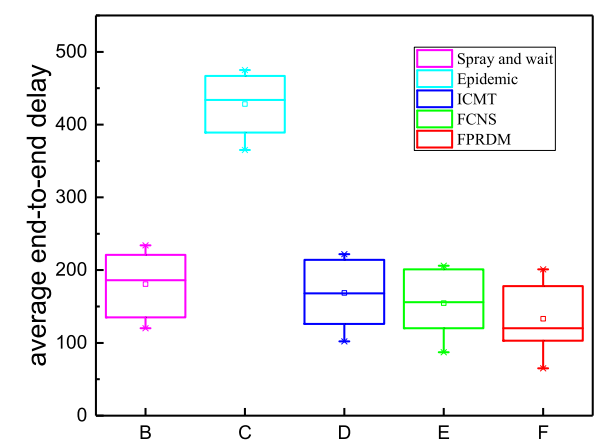

(a)

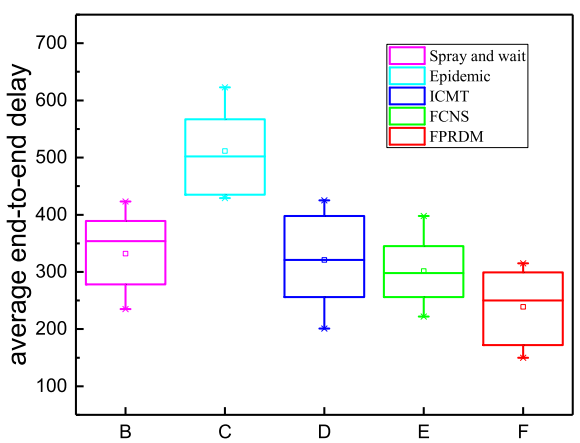

(c)

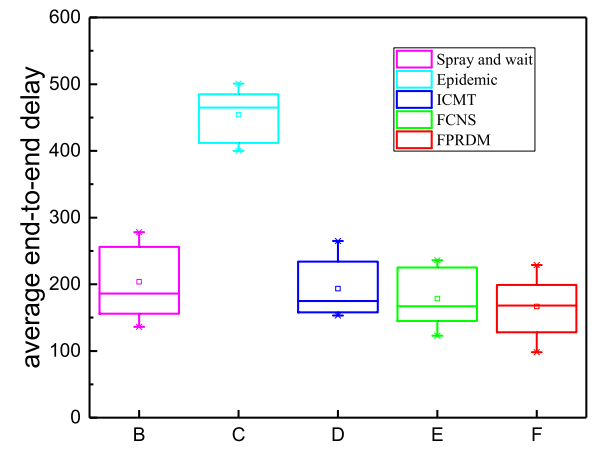

(b)

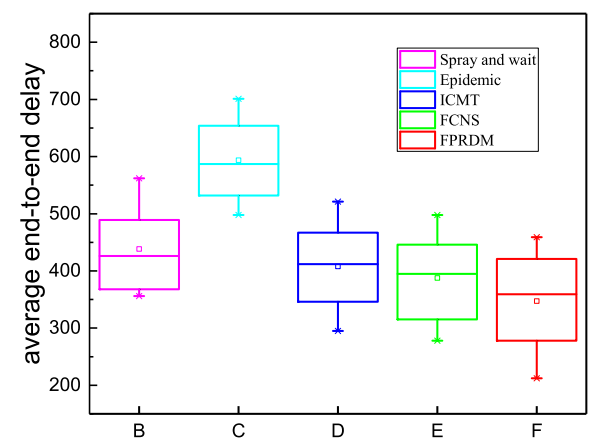

(d)

Figure 8. Performance Please comparison of average end-to-end delay with various cache spaces and various number of nodes. B: Spray and wait; C: Epidemic; D: ICMT; E: FCNS; F: FPRDM (a) N=100. (b) $N=200$. (c) $N=400$. (d) $N=600$.

As shown in Figure 8a-d, the average end-to-end delay from the FPRDM algorithm is always the lowest, while that from the FCNS method is the second lowest. It should be noted that the average end-to-end delay from FPRDM reaches the lowest value of $68 \mathrm{~s}$ when $N=100$ in Figure 8a. From Figure $8 c$, the gap of average end-to-end delay between FPRDM and FCNS reaches the maximum of $60 \mathrm{~s}$ when $N=300$, which indicates that the fuzzy control strategy of the FPRDM scheme is superior to that of the FCNS algorithm. To be specific, the FCNS algorithm focuses on node similarity, which is a flexible metric due to the changes in nodes attributes; while the FPRDM scheme considers the fuzzy data transmission relationship between nodes through their social online features, which can be converted into a digitized index and utilized to make a more reasonable data transmission decisions in OSNs. Besides, with the application of the mechanisms of node cooperation and cache management, the ICMT algorithm is able to maximally and rationally utilize the buffer memory space of each 
node , and more importantly, tremendously improving the efficiency of the data dissemination and simultaneously decreasing the average end-to-end delay.

Finally, we explore the performance comparison of the network overhead from the five routing schemes with various cache spaces and various number of nodes. As demonstrated in Figure 9, when the buff memory space of nodes constantly increases from 10 to $40 \mathrm{Mb}$, the network overhead from each algorithm declines dramatically. On the contrary, when the number of nodes in the communication area continually increases, the network overhead of each algorithm slowly goes down. As exhibited in Figure 9, the network overhead of these algorithms could be ranked from high to low as: Spray and Wait, Epidemic, ICMT, FCNS, and FPRDM. Specially, the network overhead of the FPRDM algorithm is always the lowest among these methods, presenting an average network overhead of 150 .

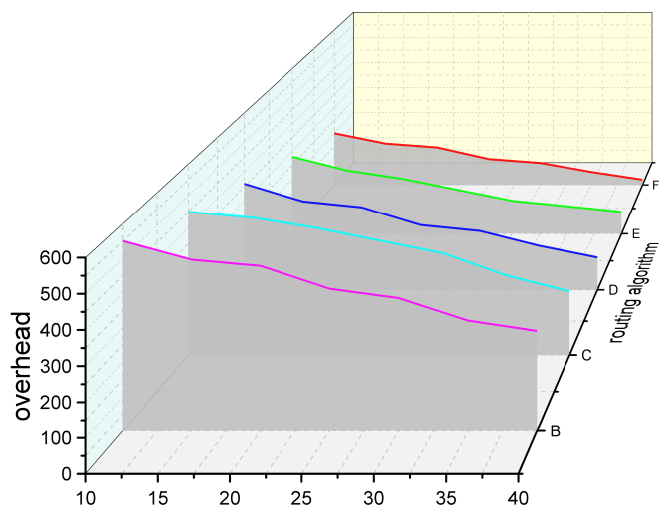

(a) Cache (Mb)

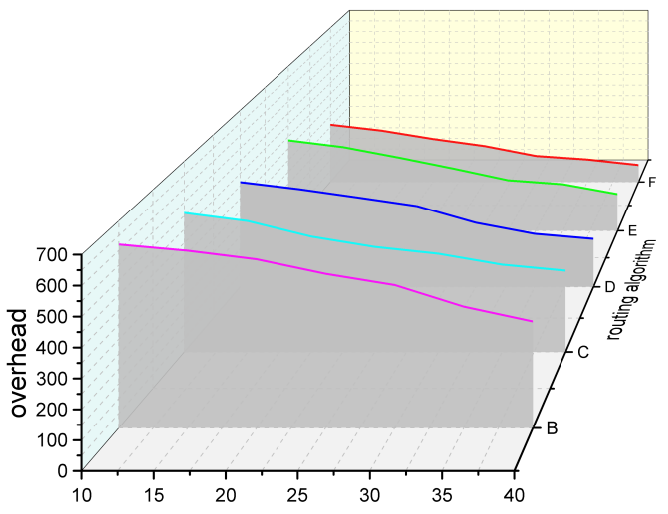

(c) Cache (Mb)

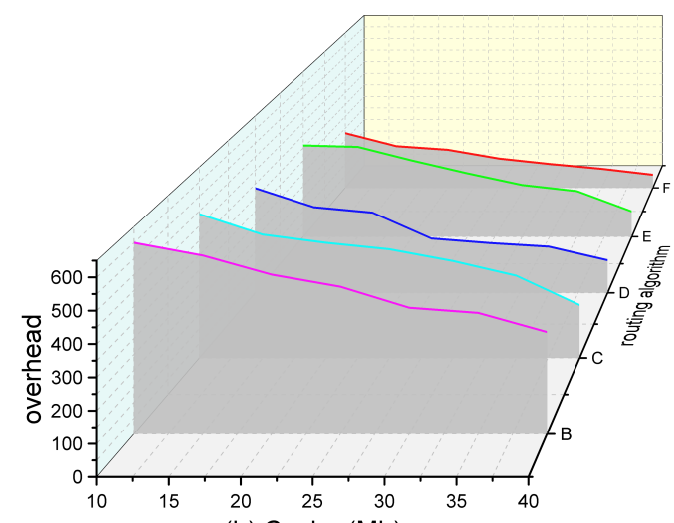

(b) Cache (Mb)

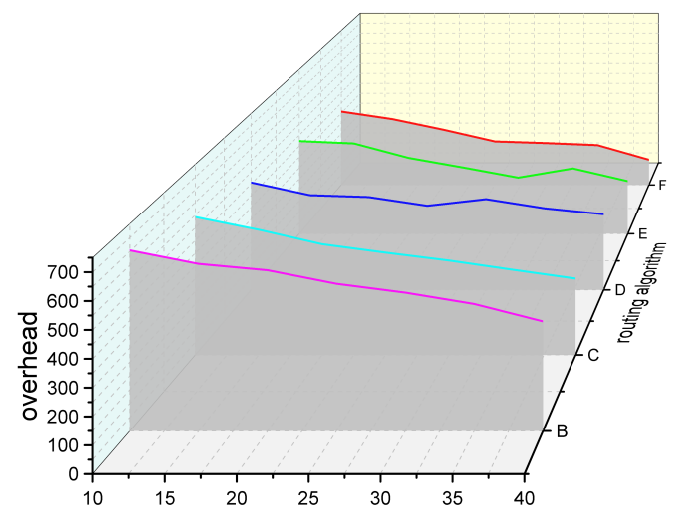

(d) Cache (Mb)

Figure 9. Performance comparison of network overhead with various cache spaces and various number of nodes. B: Spray and wait; C: Epidemic; D: ICMT; E: FCNS; F: FPRDM. (a) $N=100$. (b) $N=200$. (c) $N=400$. (d) $N=600$.

From Figure 9a, when $N=100$ and $C=40 \mathrm{Mb}$, the network overhead of the FPRDM scheme reaches the minimum value of 50, which means that this strategy adopts fuzzy inference theory to effectively control the number of message replications and simultaneously reduce the number of relay nodes. From Figure $9 \mathrm{~d}$, when $N=600$ and $C=10 \mathrm{Mb}$, the network overhead from the Spray and Wait approach reaches the maximum approximately 660 . When selecting a suitable relay node, the Epidemic routing algorithm adopts the flooding model to widely spread data packages in the network. ICMT, Spray and Wait, and FCNS employ data management, encountering history, and fuzzy control to make a more accurate decision on message routing and forwarding, respectively. This is because message replications are widely propagated in the Spray phase, while in the next phase the number of messages is tightly controlled, thereby resulting in an increase in the number of hops. Besides, the network overhead from ICMT and FCNS routing algorithms is in the middle level all the 
time, because some effective message controlling strategies such as information management, fuzzy routing selection have been adopted.

To sum up, the experimental results demonstrate the proposed scheme reduces the network delay and the overhead ratio, and increases the delivery ratio as compared with several typical and latest routing protocols. Especially, in the best case, the proposed scheme presents an average delivery ratio of 0.8 , reduces the average end-to-end delay by nearly $45 \%$ as compared with the Epidemic routing protocol, and lowers the network overhead by about $75 \%$ as compared to the Spray and Wait routing algorithm.

\section{Conclusions}

In this study, for investigating the problem of data transmission in opportunistic mobile social networks, we propose and develop an adaptive routing-forwarding control scheme based on fuzzy recognition and decision-making model (FPRDM), which contains three interlocking steps: fuzzy recognition for node classification, weight adjustment for social features, and the inference process for the fuzzy relationship between node profile and data transmission. In an opportunistic mobile social network, message users carrying smart mobile devices randomly move around within a certain communication area, so they could be identified and located by several crucial and representative social attributes. After the social characteristics of mobile users have been formalized from context information to digital eigenvector, the obtained quantization vector is considered as a fuzzy input and will be utilized to determine the corresponding fuzzy subset and membership degree. Based on the training results from the improved AHP testing in a real dataset, the FPRDM approach allocates a suitable weight to each social characteristic of message user and determines three different grades of membership functions (high, medium, and low) for the quantization vector. A high membership degree between two nodes indicates that there is a relatively high social similarity between them and they are more likely to belong to the same online community. On the contrary, a low membership between a pair of message users suggests that they do not communicate with each other frequently and there are fewer opportunities for message routing and forwarding between them. Afterwards, on the basis of the application of a defuzzfier component, the fuzzy relationship between social attribute and node recognition could be translated into a specific transmission priority value, which is regarded as a significant reference for message routing and forwarding in the networks. To evaluate the optimization performance of the FPRDM algorithm, simulation results demonstrate that it dramatically reduces the end-to-end delay and the network overhead, and slightly enhances the delivery ratio as compared with the other four routing strategies.

In future studies, we will work on the information fusion of more social features of mobile users in OSNs so that some more credible decisions on data dissemination could be provided, and more importantly, constructing an effective routing security protocol for tackling the issue of privacy protection in the data transmission process.

Author Contributions: L.Z., Y.Z., H.S., X.S. and S.L. conceived the idea of the paper. L.Z., Y.Z., H.S., K.-S.H., X.S. and S.L. designed and performed the experiments; H.S., K.-S.H., X.S. and S.L. analyzed the data; K.-S.H., X.S. and S.L. contributed reagents/materials/analysis tools; L.Z. wrote and revised the paper.

Funding: This work is supported by Civil Aircraft Project under Grant No. XJ-2015-D-76, and the major and key project of the Shaanxi Province key research and development plan under Grant No. 2016MSZD-G-8-1.

Conflicts of Interest: The authors declare that they have no competing interests. 


\section{Abbreviations}

The following abbreviations are used in this manuscript:

$\begin{array}{ll}\text { OSNs } & \text { opportunistic social networks } \\ \text { DTNs } & \text { delay tolerant networks } \\ \text { SNS } & \text { social network service } \\ \text { AHP } & \text { analytic hierarchy process } \\ \text { EpSoc } & \text { a flooding-based social-based routing protocol } \\ \text { Tanh } & \text { tanhyperbolic function } \\ \text { Markov } & \text { markov Andrey chain } \\ \text { CoA } & \text { center of area } \\ \text { MoM } & \text { mean of maximum } \\ \text { ONE } & \text { opportunistic networking environment } \\ \text { FPRDM } & \text { an adaptive control scheme based on intelligent fuzzy decision-making system } \\ \text { FCNS } & \text { fuzzy routing-forwarding algorithm exploiting comprehensive node similarity } \\ \text { ICMT } & \text { information cache management and data transmission algorithm }\end{array}$

\section{References}

1. Dede, J.; Forster, A.; Hernandez-Orallo, E.; Herrera-Tapia, J.; Kuladinithi, K.; Kuppusamy, V.; Manzoni, P.; bin Muslim, A.; Udugama, A.; Vatandas, Z. Simulating Opportunistic Networks: Survey and Future Directions. IEEE Commun. Surv. Tutor. 2017, 20, 1547-1573. [CrossRef]

2. Halikul, L.; Mohamad, A. EpSoc: Social-Based Epidemic-Based Routing Protocol in Opportunistic Mobile Social Network. Mob. Inf. Syst. 2018, 2018, 1-8.

3. Bulut, E.; Wang, Z.; Szymanski, B.K. Cost-Effective Multiperiod Spraying for Routing in Delay-Tolerant Networks. IEEE/ACM Trans. Netw. 2010, 18, 1530-1543. [CrossRef]

4. Ali, I.; Chang, R.Y.; Hsu, C.H. SOQAS: Distributively Finding High-Quality Answerers in Dynamic Social Networks. IEEE Access 2018, 6, 55074-55089. [CrossRef]

5. Pirozmand, P.; Wu, G.; Jedari, B.; Xia, F. Human mobility in opportunistic networks: Characteristics, models and prediction methods. J. Netw. Comput. Appl. 2014, 42, 45-58. [CrossRef]

6. Liu, K.; Chen, Z.; Wu, J.; Wang, L. FCNS: A fuzzy routing-forwarding algorithm exploiting comprehensive node similarity in opportunistic social networks. Symmetry 2018, 10, 338. [CrossRef]

7. Ge, X.; Ye, J.; Yang, Y.; Li, Q. User Mobility Evaluation for 5G Small Cell Networks Based on Individual Mobility Model. IEEE J. Sel. Areas Commun. 2016, 34, 528-541. [CrossRef]

8. Fang, D.; Qian, Y.; Hu, R.Q. Security for 5G Mobile Wireless Network. IEEE Access 2018, 6, 4850-4874. [CrossRef]

9. Wu, J.; Chen, Z.; Zhao, M. Information cache management and data transmission algorithm in opportunistic social networks. Wirel. Netw. 2018, 8,1-12. [CrossRef]

10. Mumtaz, S.; Al-Dulaimi, A.; Frascolla, V.; Hassan, S.A.; Dobre, O.A. Guest Editorial Special Issue on 5G and Beyond-Mobile Technologies and Applications for IoT. IEEE Internet Things J. 2019, 6, 203-206. [CrossRef]

11. Novak, E.; Tang, Z.; Li, Q. Ultrasound Proximity Networking on Smart Mobile Devices for IoT Applications. IEEE Internet Things J. 2019, 6, 399-409. [CrossRef]

12. Bangerter, B.; Talwar, S.; Arefi, R.; Stewart, K. Networks and devices for the 5G era. IEEE Commun. Mag. 2014, 52, 90-96. [CrossRef]

13. Liu, K.; Chen, Z.; Wu, J.; Xiao, Y.; Zhang, H. Predict and Forward: An Efficient Routing-Delivery Scheme Based on Node Profile in Opportunistic Networks. Future Internet 2018, 10, 74. [CrossRef]

14. Yan, Y.; Chen, Z.; Wu, J.; Wang, L.; Liu, K.; Wu, Y. Effective Data Transmission Strategy Based on Node Socialization in Opportunistic Social Networks. IEEE Access 2019, 7, 22144-22160. [CrossRef]

15. Kafaie, S.; Chen, Y.; Dobre, O.A.; Ahmed, M.H. Joint Inter-Flow Network Coding and Opportunistic Routing in Multi-Hop Wireless Mesh Networks: A Comprehensive Survey. IEEE Commun. Surv. Tutor. 2018, 20, 1014-1035. [CrossRef]

16. Wang, L.L.; Chen, Z.G.; Wu, J. Vehicle trajectory prediction algorithm in vehicular network. Wirel. Netw. 2018, 3, 1-14. [CrossRef] 
17. Luo, J.; Hu, J.; Wu, D.; Li, R. Opportunistic Routing Algorithm for Relay Node Selection in Wireless Sensor Networks. IEEE Trans. Ind. Inform. 2015, 11, 112-121. [CrossRef]

18. Prodhan, A.T.; Das, R.; Kabir, H.; Shoja, G.C. TTL based routing in opportunistic networks. J. Netw. Comput. Appl. 2011, 34, 1660-1670. [CrossRef]

19. Bapu, B.R.T.; Gowd, L.C.S. Link Quality Based Opportunistic Routing Algorithm for QOS: Aware Wireless Sensor Networks Security. Wirel. Pers. Commun. 2017, 97, 1563-1578. [CrossRef]

20. Choumas, K.; Syrigos, I.; Korakis, T.; Tassiulas, L. Video aware Multicast Opportunistic Routing over 802.11 two-hop mesh networks. IEEE Trans. Veh. Technol. 2017, 66, 8372-8384. [CrossRef]

21. Radunovic, B.; Gkantsidis, C.; Key, P.; Rodriguez, P. Toward Practical Opportunistic Routing With Intra-Session Network Coding for Mesh Networks. IEEE/ACM Trans. Netw. 2010, 18, 420-433. [CrossRef]

22. Borrego, C.; Sánchez-Carmona, A.; Li, Z.; Robles, S. Explore and Wait: A composite routing-delivery scheme for relative Profile-casting in Opportunistic Networks. Comput. Netw. 2017, 123, 51-63. [CrossRef]

23. Li, N.; Martinez-Ortega, J.F.; Diaz, V.H.; Fernandez, J.A.S. Probability Prediction-Based Reliable and Efficient Opportunistic Routing Algorithm for VANETs. IEEE/ACM Trans. Netw. 2018, 26, 1-15. [CrossRef]

24. Li, J.; Jia, X.; Lv, X.; Han, Z.; Liu, J.; Hao, J. Opportunistic routing with data fusion for multi-source wireless sensor networks. Wirel. Netw. 2019, 25, 3103-3113. [CrossRef]

25. Zeng, Y.; Chen, G.; Li, K.; Zhou, Y.; Zhou, X.; Li, K. M-Skyline: Taking sunk cost and alternative recommendation in consideration for skyline query on uncertain data. Knowl.-Based Syst. 2019, 163, $204-213$. [CrossRef]

26. Chen, G.; Lu, Y.; Meng, Y.; Li, B.; Tan, K.; Pei, D.; Cheng, P.; Luo, L.; Xiong, Y.; Zhao, Y.; et al. FUSO: Fast Multi-Path Loss Recovery for Data Center Networks. IEEE/ACM Trans. Netw. 2018, 26, 1-14. [CrossRef]

27. Trifunovic, S.; Kouyoumdjieva, S.T.; Distl, B.; Pajevic, L.; Karlsson, G.; Plattner, B. A Decade of Research in Opportunistic Networks: Challenges, Relevance, and Future Directions. IEEE Commun. Mag. 2017, 55, 168-173. [CrossRef]

28. Wang, Y.; Liu, Y.; Zhang, J.; Ye, H.; Tan, Z. Cooperative Store-Carry-Forward Scheme for Intermittently Connected Vehicular Networks. IEEE Trans. Veh. Technol. 2017, 66, 777-784. [CrossRef]

29. Liu, K.; Chen, Z.; Wu, J.; Tan, Y.; Wang, L.; Yan, Y.; Zhang, H.; Long, J. Big Medical Data Decision-Making Intelligent System Exploiting Fuzzy Inference Logic for Prostate Cancer in Developing Countries. IEEE Access 2019, 7, 2348-2363. [CrossRef]

30. Nag, K.; Helal, M. Multicriteria Inventory Classification of Diabetes Drugs Using a Comparison of AHP and Fuzzy AHP Models. In Proceedings of the 2018 IEEE International Conference on Industrial Engineering and Engineering Management (IEEM), Bangkok, Thailand, 16-19 December 2018; pp. 1456-1460.

31. Conti, M.; Giordano, S.; May, M.; Passarella, A. From opportunistic networks to opportunistic computing. IEEE Commun. Mag. 2010, 48, 126-139. [CrossRef]

32. Yang, Y.; Zhao, H.; Ma, J.; Han, X. Social-aware data dissemination in opportunistic mobile social networks. Int. J. Mod. Phys. C 2017, 28, 1750115. [CrossRef]

33. Xu, Q.; Su, Z.; Zhang, K.; Ren, P.; Shen, X.S. Epidemic Information Dissemination in Mobile Social Networks With Opportunistic Links. IEEE Trans. Emerg. Top. Comput. 2015, 3, 399-409. [CrossRef]

34. Li, N.; Martinez-Ortega, J.F.; Díaz, V.H. Cross-layer and Reliable Opportunistic Routing Algorithm for Mobile Ad Hoc Networks. IEEE Sens. J. 2018, 18, 5595-5609. [CrossRef]

35. Bródka, P.; Kazienko, P. Multilayer Social Networks. In Encyclopedia of Social Network Analysis and Mining; Springer: New York, NY, USA, 2017.

36. Magnani, M.; Rossi, L. The ML-Model for Multi-layer Social Networks. In Proceedings of the 2011 International Conference on Advances in Social Network Analysis and Mining, Kaohsiung, Taiwan, 25-27 July 2011.

37. Chen, Q.; Zhang, Q.; Niu, Z. A Graph Theory Based Opportunistic Link Scheduling for Wireless Ad Hoc Networks. IEEE Trans. Wirel. Commun. 2009, 8, 5075-5085. [CrossRef]

38. Hassan, S.A.; Ingram, M.A. A Quasi-Stationary Markov Chain Model of a Cooperative Multi-Hop Linear Network. IEEE Trans. Wirel. Commun. 2011, 10, 2306-2315. [CrossRef]

39. Wenli, F.; Zhigang, L.; Ping, H. Identifying node importance based on information entropy in complex networks. Phys. Scr. 2013, 88, 065201. [CrossRef]

40. Dutu, L.; Mauris, G.; Bolon, P. A Fast and Accurate Rule-Base Generation Method for Mamdani Fuzzy Systems. IEEE Trans. Fuzzy Syst. 2018, 26, 715-733. [CrossRef] 
41. Li, J.; Wang, Z.; Shen, Y.; Wang, Y. Interval Observer Design for Discrete-Time Uncertain Takagi-Sugeno Fuzzy Systems. IEEE Trans. Fuzzy Syst. 2019, 27, 816-823. [CrossRef]

42. Pak, J.; Song, Y.T. Health Capability Maturity Model: Person-centered approach in Personal Health Record System. In Proceedings of the Americas Conference on Information Systems, San Diego, CA, USA, 11-13 August 2016. 